
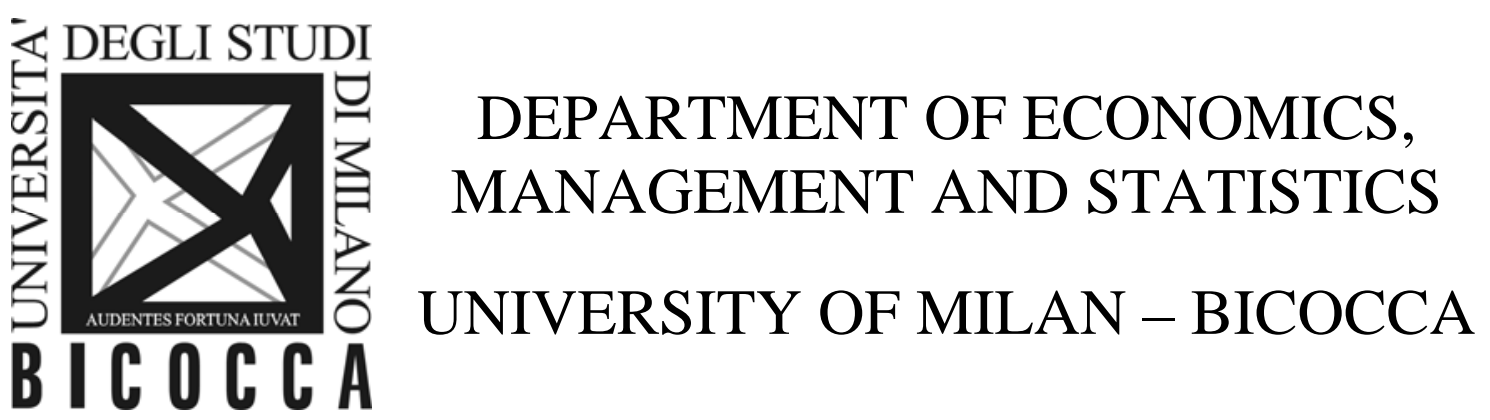

DEMS WORKING PAPER SERIES

\title{
Do The Right Thing. A comparison of politicians' incentives across constitutional systems
}

Michela Cella, Giovanna Iannantuoni, Elena Manzoni

No. 290 - January 2015

Dipartimento di Economia, Metodi Quantitativi e Strategie di Impresa Università degli Studi di Milano - Bicocca 


\title{
Do The Right Thing.
}

\section{A comparison of politicians' incentives across constitutional systems*}

\author{
Michela Cella $^{\dagger} \quad$ Giovanna Iannantuoni $^{\ddagger} \quad$ Elena Manzoni ${ }^{\S}$
}

January 7, 2015

\begin{abstract}
Constitutional structures shape politicians' behaviour and hence policy outcomes through the different incentive schemes at work. In this paper we analyse these mechanisms in parliamentary and presidential systems. Such a comparison is carried over by analysing how the two systems are able to select the efficient policy in presence of asymmetric information. The constitutional structures differ in that the policy proposal in parliamentary democracies is observable and confidence-dependent. The main findings suggest that the parliament responds to the incentive scheme better in presidential systems due to less uncertainty that legislators face over their term limit. However, the parliamentary system induces the executive to behave more efficiently due to selection and disciplining effects.

Keywords: presidential system, parliamentary system, confidence vote, hierarchical accountability
\end{abstract}

JEL Classification: C72, D72

${ }^{*}$ The authors gratefully acknowledge financial support from PRIN (2012-2015) " New approaches to political economics : positive political theories, empirical evidence and experiments in laboratory". Useful comments were received by: Leo Ferraris and Ascensión Andina-Díaz.

${ }^{\dagger}$ Department of Economics, Management and Statistics, University of Milan-Bicocca. E-mail: michela.cella@unimib.it

${ }^{\ddagger}$ Department of Economics, Management and Statistics, University of Milan-Bicocca. E-mail: giovanna.iannantuoni@unimib.it

${ }^{\S}$ Department of Economics, Management and Statistics, University of Milan-Bicocca. E-mail: elena.manzoni@unimib.it 


\section{Introduction}

In every democracy the choice and implementation of a policy are characterised by the interplay of the executive and legislative bodies. In this paper we study how different constitutional structures, presidential and parliamentary, affect the incentives of politicians in presence of asymmetric information.

The debate over the relative merits of parliamentary and presidential systems has been present in the economics and political science literature for a very long time. In the second half of the past century the issue has been dissected in many ways but still, in spite of the numbers of pages written, we have no consensus on which system has to be preferred.

We follow the seminal works of Persson and Tabellini [2002, 2005] that showed how institutional setups have a relevant impact in shaping economic policies, but we take a step further and look inside the mechanism through which new laws see the light and become effective and study how the two systems perform in terms of efficient policy selection.

Specifically, we compare presidential versus parliamentary systems through the following two-period setup. The government is defined by an executive body, represented by a single player, and by a legislative body, represented by a parliament composed of $L$ members. At the beginning of the game each player observes his type (i.e. policy/office-oriented). In the first period politicians observe the true state of the world while voters remain uninformed. At this point the executive proposes a policy that has to be approved by majority in the parliament. At the end of the first period also voters observe the true state of the world, update their beliefs and then period two occurs analogously. The first difference between the two systems is the presence of the confidence vote as a key constitutional ingredient of the parliamentary system. The main implication of the confidence requirement is that if the policy proposed by the executive is rejected, new elections are called for both government bodies. This allows the parliamentary system to replace bad politicians even before the natural conclusion of the legislature; in turn though, it makes the system also very sensitive to the incentives of legislators who may have private agendas themselves and not act in the interest of voters. The other key difference between the two constitutional frameworks is that in the presidential system voters observe only the implemented policy, while in the parliamentary one they observe also the policy proposed by the 
executive.

In our model the presidential system always selects the efficient policy, therefore outperforming the parliamentary one. The force driving this result is the perfect information of the parliament that corrects, by voting no, any attempt at inefficient behaviour by a, possibly, office-oriented executive. The fact that the length of the legislature does not depend on the approval of the executive's proposal allows the parliament to vote for the implementation of the efficient policy regardless of the composition of the legislative body. Even office-oriented members of the parliament vote for the efficient policy to maximise their end-of-period reputation in the eye of the voters who, unlike the legislators, do not observe the true state of the world and update their beliefs about the type of politicians by observing only the implemented policy. In a parliamentary system instead, office-oriented legislators have a strong incentive to approve anything proposed by the executive just to avoid early elections and stay in office until the natural end of the legislature and, as a consequence, the inefficient policy will be implemented with positive probability. The presence of the confidence vote therefore generates a distortion in the incentives of office-oriented legislators. On the other side though, the confidence vote allows policy-oriented members of parliament to replace an office-oriented executive before the legislature is over. It follows that the quality of the proposal in the second period improves because officeoriented governments are voted out of office more often. This is a selection effect that improves the executive performance in the parliamentary system in the final period with respect to the presidential one. The confidence vote has also a disciplining effect in the first period, if the cost of implementing the efficient policy is not too high, as the executive may propose the efficient policy for fear of being voted against and face early elections.

On top of this, the observability of the executive's proposal in the parliamentary system improves the behaviour of the executive in the second period, when new elections are certain and the executive wants to maximise his end-of-period reputation with voters.

In our model the positive incentives in the parliamentary system are completely offset by the perfect behaviour of the parliament in the presidential one that is driven by the symmetric information among politicians about the state of the world. At the end of the paper we provide a simple example where the parliament is not perfectly informed about the state of the world 
and votes according to an informative signal. In this case the presidential system no longer achieves full efficiency, as the parliament may not correct the misbehaviour of the executive due to an incorrect signal. We show that in this case the parliamentary system may outperform the presidential one and select the efficient policy with higher probability.

Our work is related, as mentioned above, to the literature that links constitutional design and economic policy that began with Persson and Tabellini $[2002,2005]$ and to the literature on incentives in political economy (see for example Besley [2007]). Like them we fit in what Elgie [2005] calls the "third wave" of studies that have addressed the relative merits of presidential and parliamentary systems.

More precisely, the idea that a good way to judge a political system is its ability to select the efficient policy comes from Besley and Coate [1998], where, in a different setup, they identify a political failure as the inability to undertake a potentially Pareto improving public investment with the available policy instrument.

Another related work is Diermeier and Vlaicu [2011] who study how constitutional features influence political behaviour in terms of legislative success (passing of bills proposed by the executive) and they show that the confidence vote (that may send everybody home) is the critical feature that may explain the different performance of a parliamentary and presidential system in terms of legislative success. Also Becher [2012] believes that differences in executive-legislative institutions shape the policymaking capacity of the two systems and identifies the dissolution power of the executive in the parliamentary framework as the force that leads to higher spending in parliamentary systems versus presidential ones.

Our hierarchical agency structure is related to the one in Vlaicu [2008] and Vlaicu and Whalley [2013] where they study accountability in government under different hierarchical controls without comparing different constitutions. Also in their models politicians have private information about their type and voters and other politicians update their beliefs by observing their institutional activities.

The structure of the papers is as follows: Section 2 describes the elements of the model, Sections 3 presents the equilibrium analysis, Section 4 contains an example where the assembly is not fully informed, Section 5 briefly concludes. All proofs are in the Appendix. 


\section{The model}

We introduce a political economy framework of policy formation resulting from the interplay between an executive and a legislative body. In order to compare the two institutional setups, i.e. a presidential and a parliamentary system, we present the following two-period structure in which voters (passive) and politicians face an uncertain policy environment.

Policy environment. We assume a two-period model in which every period $t=1,2$ is characterised by a state of the world $s_{t} \in\left\{s_{A}, s_{B}\right\}$. States are equally likely, in that $\mathbb{P}\left[s_{t}=s_{k}\right]=\frac{1}{2}$ for $k=A, B$, and $t=1,2$, and independently distributed across periods. In every period a public good $g_{t} \in\{A, B\}$ has to be produced where we assume that good $A \operatorname{costs} c_{A} \in(0,1)$ and we normalize the cost of $B$ to zero. In every period the amount of resources the executive can use either for the production of the public good or for private consumption is one.

The policy is determined by the behaviour of the executive and the parliament: The executive proposes a policy $g_{t}^{e} \in\{A, B\}$ and the parliament votes on it. If the parliament rejects the proposal a status-quo policy $g^{0}=A$ is implemented. ${ }^{1}$

Voters. In this context voters are passive players in that we do not model the voting stage. Hence, the electorate is composed by $N$ homogeneous voters; with a slight abuse of notation we define the per-period utility $u\left(g_{t}, s_{k}\right)$ such that for every $t=1,2$ and $k=A, B$ :

$$
u\left(g_{t}, s_{k}\right)=\left\{\begin{array}{lc}
1, & \text { if } g_{t}=k \\
0, & \text { otherwise }
\end{array}\right.
$$

Hence, the efficient policy is $g^{*}\left(s_{t}\right)$, where:

$$
g^{*}\left(s_{t}\right)= \begin{cases}A, & \text { if } s_{t}=s_{A} \\ B, & \text { if } s_{t}=s_{B} .\end{cases}
$$

We assume that voters perfectly observe $s_{1}$ only at the end of period one.

\footnotetext{
${ }^{1}$ We focus on $g^{0}=A$ because this is the most interesting case, as $A$ is the costly good.
} 
Executive. The executive body cares about rents, which in our framework are given by the amount of resources not invested in the public good (i.e. $1-c_{g_{t}}$ ) and may also care directly about the implemented policy. Following the political economy literature let us define the executive policy-oriented if he cares about the implemented policy, and office-oriented if not. Being policy/office-oriented is private information. To be more specific, the executive is characterized by a privately observed type $\theta^{e} \in\{0,1\}$ where $\theta^{e}=1$ means a policy-oriented executive. We denote the probability of being policy-oriented $\mathbb{P}\left[\theta^{e}=1\right]=\gamma$.

The executive's utility function is:

$$
U^{e}=1-c_{g_{1}}+\theta^{e} u\left(g_{1}, s_{1}\right)+\pi\left(1-c_{g_{2}}+\theta^{e} u\left(g_{2}, s_{2}\right)+\varepsilon \widehat{\theta}^{e}\right) .
$$

where $u\left(g_{t}, s_{t}\right)$ is the per-period utility from policy experienced in case the executive is policy-oriented. The interpretation is that the executive cares about the policy, if $\theta^{e}=1$, exactly in the same way as voters do. The second part of the utility is weighted by the probability of being in power in period two, $\pi$. Let $\widehat{\theta}^{e}$ be the ex-post voters' belief on the probability that the executive is policy-oriented, and let $\varepsilon \in\left[0, c_{A}\right]$. The term $\varepsilon \widehat{\theta}^{e}$ represents in a reduced form the executive's concerns about future elections. Intuitively, the parametric assumption on $\varepsilon$ ensures that, in period two, a policy-oriented executive cares more about the implemented policy than about his final reputation.

The executive observes the state of the world in every period.

Parliament. The legislative body has to approve or reject the executive's policy proposal in each period. The parliament is composed of $L$ (odd) legislators, $l=1, \ldots, L$. Analogously to the executive body, every legislators can be policy- or office-oriented and this characteristic is his private information. Hence, each legislator privately observes his type $\theta^{l} \in\{0,1\}$ where $\theta^{l}=1$ means a policy-oriented legislator. The probability of being policy-oriented is $\mathbb{P}\left[\theta^{l}=1\right]=\gamma$, and types are independent across members. We are therefore assuming that both executive and legislative posts are filled with politicians drawn from the same pool.

The utility function of legislator $l$ is:

$$
U^{l}=\left(1-\theta^{l}\right) R+\theta^{l} u\left(g_{1}, s_{1}\right)+\pi\left(\left(1-\theta^{l}\right) R+\theta^{l} u\left(g_{2}, s_{2}\right)+\varepsilon \widehat{\theta}^{p}\right),
$$


where $R \geq \varepsilon$ is the office-holding part of the legislator's utility while $u\left(g_{t}, s_{t}\right)$ is the policy component. Office-oriented legislators care about being in office, and their rent does not depend on the implemented policy. Policy-oriented legislators, instead, only draw utility from the implementation of the efficient policy. The parametric assumption on $R$ ensures that in the first period the office-holding concerns are stronger than concerns for reputation for the office-oriented legislators. Moreover, as before, $\varepsilon \widehat{\theta}^{p}$ represents in a reduced form the legislator's concerns about future elections, where $\widehat{\theta}^{p}$ is the expost voters' belief on the probability that the majority of the parliament is policy-oriented.

Each legislator observes the state of the world in every period.

The institutional framework. Both systems are analyzed over two periods. In the presidential system at $t=0$ each player observes his private type, at $t=1$ the politicians observe the state of the world $s_{1}$. Then the executive makes a policy proposal and, if it is different from the status quo policy, the parliament votes to accept or reject it. Notice that if $g_{t}^{e}=g^{0}=A$ then voting over the proposal is irrelevant. At the end of period one, voters observe the state of the world $s_{1}$ and, given the implemented policy $g_{1}$, they update their beliefs on the type of the executive and on the type of the parliament. In period two politicians observe $s_{2}$ and jointly determine the policy, as described in period one. Voters, who do not observe $s_{2}$, update their beliefs on the executive and the parliament given $g_{2}$.

In the parliamentary system at $t=0$ each player observes his private type, at $t=1$ the politicians observe the state of the world $s_{1}$. Then the executive makes a policy proposal and, if it is different from the status quo policy, the parliament votes to accept or reject it. If the policy is rejected a new executive $(\widetilde{E})$ and a new parliament $(\widetilde{P})$ are elected. The new executive and the new legislators are randomly drawn from the same pool of politicians (with probability of being policy-oriented $\gamma$ ), and we assume that the probability that either the old executive or a member of the old parliament is reelected is 0 . Hence in the parliamentary system, contrary to the presidential one, voters may also infer the policy proposal of the executive, i.e. $g_{1}^{e}$. At the end of period one, voters observe $s_{1}$ and update their beliefs given $g_{1}^{e}$ and $g_{1}$. In period two politicians (also in case of reelected bodies) observe $s_{2}$ and jointly determine the policy, as described in period one. Voters, who 
do not observe $s_{2}$, update their beliefs on the executive and the parliament given $g_{2}^{e}$ and $g_{2}$.

Given the described structures, we notice that $\pi=1$ for the presidential system and $\pi \leq 1$ for the parliamentary system.

Figure 1: The Presidential system

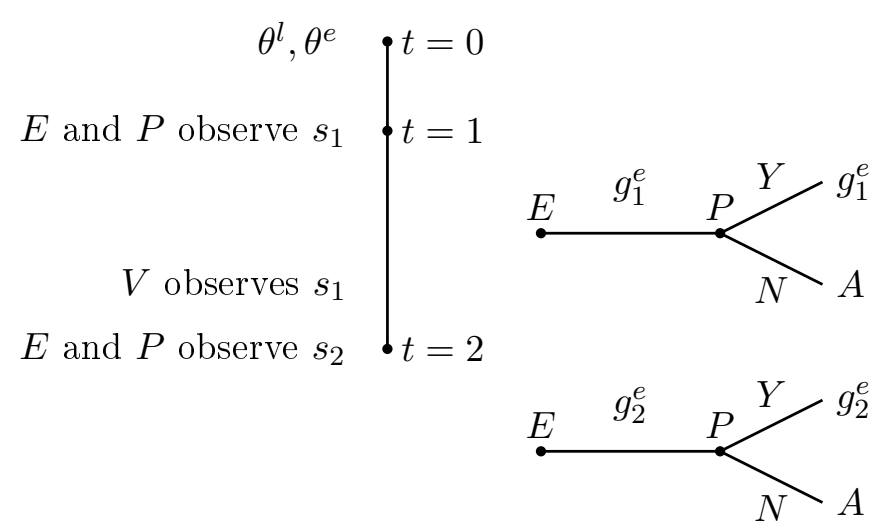

Figure 2: The Parliamentary system

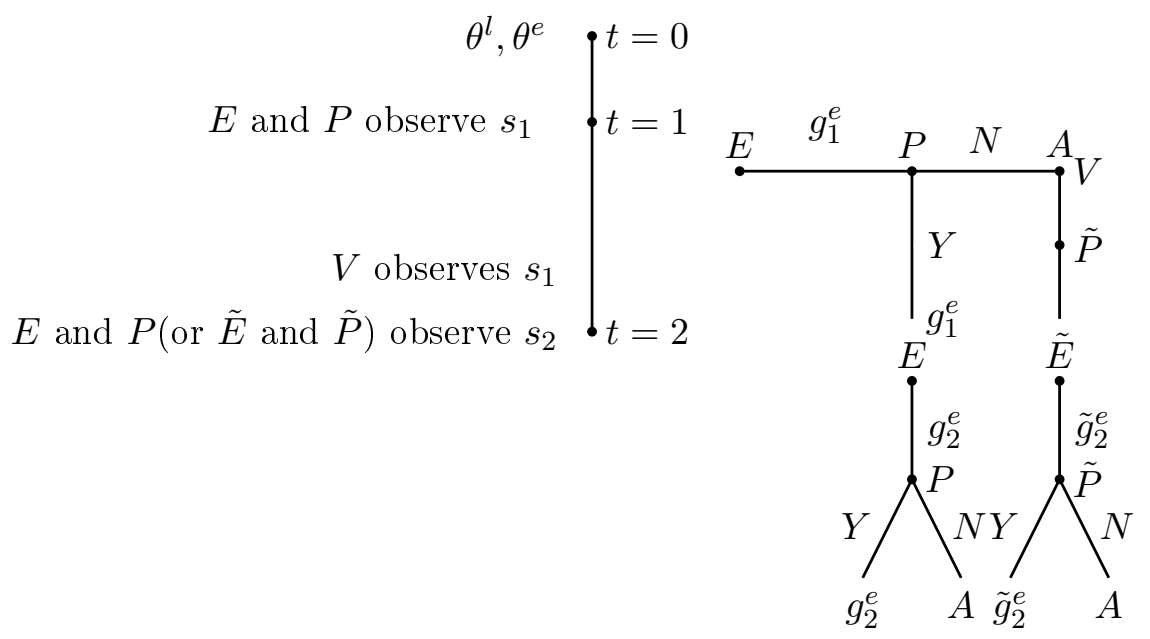




\section{Equilibrium analysis}

\subsection{The presidential system}

The main constitutional feature of the presidential system is that, given the absence of a confidence vote, the executive and the parliament hold office in both periods. This characteristic has interesting consequences for both bodies. The executive is not disciplined by the threat of a new election, hence office-oriented politicians offer the costless policy in both periods. Interestingly, the parliament, that does not control the executive through the confidence vote, induces the selection of the efficient policy outcome as a response to the following incentive scheme. On one hand, policy-oriented legislators maximise their utility by voting for the efficient policy. On the other hand, office-motivated legislators, whose utility function is $U^{l}=2 R+\varepsilon \widehat{\theta}^{p}$, have the same behaviour because they maximise the final reputation of the parliament. We summarize this intuition in the following proposition:

Proposition 1 In the presidential system we have the following pure-strategy equilibrium:

- Each legislator approves $B$ iff $s_{t}=s_{B}$;

- Both types of executive propose $B$ if $s_{t}=s_{B}$ and they are indifferent between any policy proposal if $s_{t}=s_{A}$.

Notice that even if we do not have uniqueness of the equilibrium strategies, we have uniqueness of the equilibrium outcome. As already mentioned, even in this simple framework, the parliament (who does not have the disciplining power given by the confidence vote) corrects any misbehaviour of the executive by voting against inefficient proposals. The power of the parliament in this context derives from: the undistorted incentive scheme legislators face; the ability to ascertain the efficient policy (remember that politicians observe the state of the world unlike the voters); the nature of the status quo policy, which contrasts the interests of the office-oriented executive. $^{2}$ Both types of executive are indifferent between offering the efficient

\footnotetext{
${ }^{2}$ Notice that we assumed $g^{0}=A$. If we instead assumed $g^{0}=B$ the presidential and the parliamentary systems deliver the same results, i.e. policy oriented executive would offer $g^{*}\left(s_{t}\right)$, the office-oriented executive would offer $B$, and the parliament would vote only when $A$ is proposed.
} 
policy $g^{*}\left(s_{t}\right)$ or $B$. This behaviour arises from two features of our framework: first, legislators vote for the efficient policy (as explained above); second, voters only observe the implemented policy. As a consequence the executive is indifferent between any policy proposal when $s_{t}=s_{A}$ as the implemented policy is $A$.

The above proposition has a striking effect on the welfare analysis. The efficient policy is implemented in every period and in every state of the world. Hence, the average probability of doing the right thing is one.

\subsection{The parliamentary system}

The parliamentary system differs from the presidential one in two constitutional features: in the first period policies are confidence-dependent and voters observe both the proposed and the implemented policy in every period. These characteristics shape the incentive schemes the executive and the parliament face. The parliament now has the disciplining power deriving from the confidence vote. However, this power comes at the cost of a distortion in the legislators' first-period incentives. As a matter of fact the confidence vote affects also the probability that the parliament remains in office in the second period. For the sake of simplicity we assume that both bodies face reelection in case the executive policy proposal does not pass the vote in parliament. Nevertheless, our results hold also in case this happens with a positive, but small, probability. Policy-oriented legislators maximise their utility by voting for the efficient policy in both periods. Office-oriented legislators react to the uncertainty above described by approving any policy the executive proposes in the first period. In the second period this effect vanishes hence office-oriented legislators behave efficiently. The aggregate behaviour of the parliament depends on the type of the legislators, i.e. if the majority is policy- or office-oriented. We denote by $\Gamma$ the probability that the majority of the assembly is policy-oriented, where

$$
\Gamma=\sum_{k=\frac{L-1}{2}+1}^{L}\left(\begin{array}{l}
L \\
k
\end{array}\right) \gamma^{k}(1-\gamma)^{L-k} .
$$

In this constitutional framework, the executive may face a trade-off between implementing the desired policy and remaining in power. Such a trade-off is a function of the cost of $A$ and of the quality of the parliament 
$\Gamma$. The following proposition shows the equilibrium behaviours in function of the mentioned parameters:

Proposition 2 In the parliamentary system policy-oriented legislators approve $B$ iff $s_{t}=s_{B}$ in every period, office-oriented legislators approve $B$ always in the first period and iff $s_{t}=s_{B}$ in the second period; the executive behaves according to the following two pure-strategy equilibria:

-Equilibrium 1: a policy-oriented executive proposes the efficient policy, an office-oriented executive proposes $B$ in the first period and the efficient policy in the second period if $c_{A}>\frac{2(\varepsilon+\Gamma)}{2-\Gamma}$;

-Equilibrium 2: both types of executive propose the efficient policy in the first and in the second period if $c_{A}<\frac{2(\varepsilon \gamma+\Gamma)}{2-\Gamma}$.

The above proposition shows that the parliamentary system performs like the presidential one when the cost of implementing policy $A$ is not too high, as in both systems the average probability of doing the right thing is one. Notice that in the first period this derives from the efficient behaviour of the executive despite an inefficient behaviour of the parliament (contrary to the presidential system). When the cost of implementing policy $A$ is high enough instead, both bodies behave inefficiently in the first period (for the reasons above explained) hence the average probability of doing the right thing is $\frac{3}{4}+\frac{\Gamma}{4}+\frac{\gamma}{4}(1-\Gamma)$ which is smaller than one.

\subsection{Comparison between the two systems}

The presidential system in our framework achieves the first best, in that it always induces the implementation of the efficient policy. In this respect, the presidential system outperforms the parliamentary one, that achieves the first best only when the cost of implementing policy $A$ is not too high. However, the two constitutional setups operate in a complementary way in shaping the politicians' incentive schemes.

The strength of the presidential system is that it provides an undistorted incentive scheme to the parliament. When legislators are perfectly informed, this implies that the parliament corrects any possible misbehaviour of an office-oriented executive.

On the other side, the parliamentary system is effective in improving the performance of the executive, both through a disciplining and a selection 
effect. When the first type of equilibrium arises, the quality of the proposed policy in the first period is the same as in the presidential system, as an officeoriented executive proposes $B$ in every state of the world. However, officeoriented executives are voted out of office more often than policy-oriented ones, hence the selection of the executive improves the quality of the proposed policy in the second period. If instead the cost of production of policy $A$ is low, the parliamentary system has a disciplining effect on the first period behaviour of the executive, which implies that every type of executive proposes the efficient policy in the first period.

To understand how these effects influence the policy determination process, we now consider two parametric examples. In both examples we consider the case in which there is an equal probability that politicians are policy- or office-oriented (i.e. $\gamma=\frac{1}{2}$, which also implies $\Gamma=\frac{1}{2}$ ). Under this assumption, in the presidential system the executive proposes the efficient policy with probability at least $\frac{1}{2}$ in every period; the implemented policy is always the efficient one because the parliament always votes against $B$ when $s_{t}=s_{A}$; finally, the probability that the executive is policy-oriented is $\frac{1}{2}$ in every period. We now analyse the implications of the parliamentary system in a case of high cost of production of $A$ and in a case of low cost. In both cases we assume $\varepsilon=\frac{1}{10}$.

Example 1 (high cost). Let us first consider the case in which $c_{A}=\frac{9}{10}$. In this case $c_{A}>\frac{2(\varepsilon+\Gamma)}{2-\Gamma}=\frac{4}{5}$, hence Equilibrium 1 arises in the parliamentary system. As a consequence, the probability that the executive proposes the efficient policy in the first period is $\frac{1}{4}$, as in the presidential system. The parliament, however, votes against $B$ when $s_{1}=s_{A}$ only with probability $\frac{1}{2}$, i.e. when the majority of the legislators are policy-oriented, so the probability that the efficient policy is implemented in the first period is $\frac{7}{8}<1$. Whenever the parliament votes against $B$, new elections are called for both bodies, and the newly elected politicians are policy-oriented with probability $\frac{1}{2}$. Hence, the probability that the executive is policy-oriented in the second period is $\frac{5}{8}>\frac{1}{2}$, due to this selection effect. In the second period the probability that the efficient policy is proposed is therefore $\frac{13}{16}$. In this aspect the parliamentary system outperforms the presidential one, due to the better average quality of the executive. In any case the parliament votes against an inefficient proposal with probability one, so the efficient policy is implemented in the second period. The average probability of doing the 
right thing is therefore $\frac{15}{16}<1$.

Example 2 (low cost). Let us now consider the case in which $c_{A}=\frac{3}{10}<\frac{11}{15}=\frac{2(\varepsilon \gamma+\Gamma)}{2-\Gamma}$. In this case Equilibrium 2 arises in the parliamentary system. As a consequence, every type of executive proposes the efficient policy in the first period, so that the efficient policy is implemented with probability one. Notice that this is due to a well behaved executive which is disciplined by the threat of a negative confidence vote; in this way, the parliament is never active, and the distorted incentive schemes that legislators face do not influence the policy determination process. As the same executive remains in power for both periods, the probability that the executive is policy-oriented in the second period is $\frac{1}{2}$. This implies that in the second period the efficient policy is proposed with probability $\frac{3}{4}$. However, the parliament corrects any misbehaviour in the second period, and the efficient policy is implemented with probability one.

\section{An example with asymmetric information on the state of the world.}

The examples introduced at the end of the previous section highlight the strengths and weaknesses of the two constitutional systems. As long as legislators are fully informed on the state of the world, the presidential system always induces the efficient policy due to the behaviour of the well-functioning and well-informed parliament. Hence, the parliamentary system can at most match its performance. If we relax the assumption that legislators observe $s_{t}$, however, the presidential system fails to achieve first best, and further investigation is needed to understand which constitutional system is the most efficient one. In this section we show an example in which the parliamentary system outperforms the presidential one when the parliament does not perfectly observe the state of the world.

We assume that each legislator receives a common signal $\sigma_{t}$ on the state of the world; the signal has precision $\frac{2}{3}$ and it is observed in each period before voting on the executive's policy proposal. Formally the signal is as 
follows:

$$
\begin{aligned}
& \sigma_{t}=\left\{\begin{array}{l}
s_{A} \text { with probability } \frac{2}{3}, \\
s_{B} \text { with probability } \frac{1}{3},
\end{array} \text { if } s_{t}=s_{A} ;\right. \\
& \sigma_{t}=\left\{\begin{array}{l}
s_{B} \text { with probability } \frac{2}{3}, \\
s_{A} \text { with probability } \frac{1}{3},
\end{array} \text { if } s_{t}=s_{B} .\right.
\end{aligned}
$$

Legislators (and voters) perfectly observe $s_{1}$ before the beginning of period 2 , hence legislators update their belief on the executive's type based on the true realization of the state of the world.

As in the previous examples we keep the assumption that $\gamma=\frac{1}{2}$ (which implies $\Gamma=\frac{1}{2}$ for any size of the assembly), and $\varepsilon=\frac{1}{10}$; we allow $c_{A} \in\left[\frac{3}{10}, 1\right)$, which includes both specifications of the previous examples.

\subsection{Presidential system}

The introduction of asymmetric information on the state of the world does not change the structure of the equilibrium in the presidential system. However, legislators, who do not observe $s_{t}$, behave in function of the precision of their signal. Given such precision, and the executive's equilibrium strategies, legislators find optimal to follow the signal (when it contrasts with the policy proposal) whenever the probability that the executive is policy-oriented is at most $\frac{1}{2}$, and to approve the proposal in any case when such probability is larger than $\frac{1}{2}$. This implies that legislators may vote in a different way in the first and in the second period. In the first period, given our parametric specification, they follow their signal. In the second period their behaviour depends on the belief $\widehat{\gamma}^{p}$ that legislators hold at the beginning of the second period. In particular, if the executive has signalled himself as policy-oriented $\left(\widehat{\gamma}^{p}=1\right)$, legislators approve his proposal regardless of $\sigma_{2}$.

The following proposition describes the equilibrium behaviour.

Proposition 3 In the presidential system with asymmetric information, given the assumed parametric specification, we have the following pure-strategy equilibrium:

- policy- and office-oriented legislators reject $B$ in the first period when $\sigma_{1}=$ $s_{A}$, and they reject $B$ in the second period when $\sigma_{2}=s_{A}$ and $\left(g_{1}^{e}, s_{1}\right) \neq$ $\left(A, s_{A}\right)$. 
- a policy-oriented executive proposes $g_{t}^{e}\left(s_{t}, 1\right)=g^{*}\left(s_{t}\right)$, for every period $t=1,2$;

- an office-oriented executive proposes $g_{t}^{e}\left(s_{t}, 0\right)=B$, for every period $t=$ 1,2 .

In this context the presidential system fails to achieve the first best, as legislators do not observe the state of the world but only an imprecise signal about $s_{t}$. As a consequence, the parliament, who still faces undistorted incentives, is not as effective as before in correcting the potential misbehaviour of office-oriented executives.

\subsection{Parliamentary system}

The introduction of asymmetric information on the state of the world reduces the legislators' understanding of the nature of the efficient policy also in the parliamentary system. In presence of the confidence vote this implies that, contrary to what we had before, a policy-oriented executive may be replaced if he proposes $B$ when the state is $s_{B}$ due to an incorrect signal $\sigma_{1}$. However, the parliamentary system still displays a positive selection effect, as officeoriented politicians are more likely to be replaced at the end of the first period, and, as a consequence, the average quality of the executive in the second period is higher than $\gamma$.

The equilibrium structure changes in two main aspects. First of all, the second period of the parliamentary system leads to the same equilibrium strategies as the presidential one. The main difference, however, rests in the first period behaviour. As a matter of fact an equilibrium analogous to Equilibrium 1 of Proposition 2 does not exist if policy-oriented legislators play pure-strategies. Office-oriented executives find optimal to propose $B$ always only if the probability that policy-oriented legislators reject it (when $\left.\sigma_{1}=s_{A}\right)$ is smaller than one. Notice that Equilibrium 2 of Proposition 2 does not exist anymore.

The following proposition describes the structure of the equilibrium.

Proposition 4 In the parliamentary system with asymmetric information, given the assumed parametric specification, we have the following equilibrium:

- policy-oriented legislators reject $B$ in period 1 when $\sigma_{1}=s_{A}$ with probability $x=\frac{15 c_{A}-3}{5 c_{A}+10} ;$ 
- office-oriented legislators always approve B in the first period;

- policy- and office-oriented legislators in the second period approve $B$ if $\left(s_{1}, g_{1}^{e}\right)=\left(s_{A}, A\right)$ and follow their signal otherwise;

- a policy-oriented executive proposes $g_{t}^{e}\left(s_{t}, 1\right)=g^{*}\left(s_{t}\right)$, for every period $t=1,2$;

- an office-oriented executive proposes $g_{1}^{e}\left(s_{t}, 0\right)=B$, for every period $t=$ 1,2 .

\subsection{Comparison of the two systems}

Let us now compare the welfare, in terms of the expected probability of implementing the efficient policy, in the two constitutional systems.

The presidential system has, in the first period, a probability of implementing the efficient policy of

$$
w_{1}^{\text {pres }}=\frac{1}{4}+\frac{3}{4} * \frac{2}{3}=\frac{3}{4} .
$$

This is due to the fact that, whenever $B$ is proposed, the parliament votes according to its signal, $\sigma_{1}$, which is characterised by a precision of $\frac{2}{3}$; policy $A$ is instead proposed only when it is efficient, and the executive is policyoriented, which overall happens with probability $\frac{1}{4}$. In the second period there is extra information due to the first period learning. If the first period is characterized by $\left(s_{1}, g_{1}^{e}\right)=\left(s_{A}, A\right)$, which happens with probability $\frac{1}{4}$, the legislators understand that the executive is policy-oriented and proposing the efficient policy, and approve every policy proposal; if the first period is $\left(s_{1}, g_{1}^{e}\right)=\left(s_{B}, B\right)$, which happens with probability $\frac{1}{2}$, the legislators do not learn anything from the first period behaviour, and the probability of implementing the efficient policy in the second period is again $\frac{3}{4}$; if the first period delivered $\left(s_{1}, g_{1}^{e}\right)=\left(s_{A}, B\right)$ the legislators understand that the executive is office-oriented and the expected probability of implementing the efficient policy is $\frac{2}{3}$. Hence, the overall probability of implementing the efficient policy in the second period of the presidential system is

$$
w_{2}^{\text {pres }}=\frac{1}{4}+\frac{1}{2} * \frac{3}{4}+\frac{1}{4} * \frac{2}{3}=\frac{19}{24} .
$$

The average probability of doing the right thing is therefore $W^{\text {pres }}=\frac{w_{1}^{\text {pres }}+w_{2}^{\text {pres }}}{2}=$ $\frac{37}{48}$. Notice that the presidential system performs better in the second period than in the first period, due to the learning process. 
The parliamentary system has, in the first period, a probability of implementing the efficient policy of

$$
w_{1}^{\text {parl }}=\frac{1}{4}+\frac{1}{4} * \frac{1}{2} * \frac{2}{3} x+\frac{1}{2} *\left(\frac{1}{2}+\frac{1}{2} * \frac{2}{3}+\frac{1}{2} * \frac{1}{3} *(1-x)\right)=\frac{3}{4} .
$$

This is due to the fact that when $B$ is proposed, the parliament votes according to its signal $\sigma_{1}$, with precision $\frac{2}{3}$, only when the majority of its members are policy-oriented, and approves $B$ regardless of the realization of the signal otherwise. For this particular precision of the signal the parliamentary system performs as the presidential one in the first period. In the second period, there is extra information due to the first period learning, and a higher probability that the executive is policy-oriented, due to the confidence vote in the first period. If the first period delivered $\left(s_{1}, g_{1}^{e}\right)=\left(s_{A}, A\right)$, which happens with probability $\frac{1}{4}$, the original executive is still in power and recognized as policy-oriented, and the second period probability of implementing the efficient policy is one. If the first period is characterized by $\left(s_{1}, g_{1}^{e}\right)=\left(s_{B}, B\right)$, which happens with probability $\frac{1}{2}$, the second period executive has a probability of being policy-oriented of $\frac{1}{2}$; this may happen either because the first period executive is still in power (if the assembly approves $B$ ) or because there is a new executive. Regardless of the mechanism in place, the probability of having a policy-oriented executive is $\frac{1}{2}$; hence, the probability of implementing the efficient policy in the second period is $\frac{3}{4}$. Finally, if $\left(s_{1}, g_{1}^{e}\right)=\left(s_{A}, B\right)$ two things can happen: if the legislators reject $B$, there is a new executive that is policy-oriented with probability $\frac{1}{2}$, and the probability of implementing the efficient policy in the second period is $\frac{3}{4}$; if the legislators approve $B$, they understand upon observing $s_{1}$ that the executive is office-oriented and vote according to $\sigma_{2}$ in the second period, hence the probability of implementing the efficient policy is $\frac{2}{3}$. Overall the probability of implementing the efficient policy in the second period of the parliamentary system is

$$
w_{2}^{\text {parl }}=\frac{1}{4}+\left(\frac{1}{2}+\frac{1}{4} * \frac{1}{2} * \frac{2}{3} x\right) \frac{3}{4}+\frac{1}{4}\left(1-\frac{1}{2} * \frac{2}{3} x\right) * \frac{2}{3}=\frac{1}{144} x+\frac{19}{24} .
$$

The average probability of doing the right thing in the parliamentary system is $W^{\text {parl }}=\frac{37}{48}+\frac{x}{288}$.

In this example the parliamentary system outperforms the presidential one due to the selection effect that the confidence vote has in the first period. 
Note that the asymmetric information assumption reduces the performance of both systems, as it worsens the ability of the parliament to ascertain the nature of the efficient policy. This, however, has a stronger effect on the presidential system which relies more heavily on the parliament to implement the efficient policy.

\section{Concluding remarks}

This paper analyzes the incentive schemes generated by two constitutional systems, a presidential and a parliamentary one, and their effects on the implementation of efficient policies over time.

We identify two key differences between the systems: the presence of the confidence vote and the observability of policy proposals in the parliamentary system. We find that the strength of the presidential system is that it offers undistorted incentives to legislators, thus inducing an efficient behaviour in the parliament. The structure of the parliamentary system, on the other hand, improves the quality of the proposed policy through a disciplining effect, due to the threat of a negative confidence vote, and a selection effect, as office-oriented executives are more likely to be replaced.

We prove that, when legislators are fully informed, the presidential system outperforms the parliamentary one in that the efficient policy is implemented in every state and in every period. The parliamentary system instead achieves the first best only when the costly policy $A$ is cheap, while it fails to do so when the cost of $A$ is high.

We show that the ranking between the constitutional systems may change if we reduce the quality of the information that legislators receive. We provide an example in which the parliamentary system outperforms the presidential one and we highlight the mechanisms that generate such a result.

\section{References}

[1] Besley, T. (2007) Principled Agents?: The Political Economy of Good Government, Oxford University Press.

[2] Besley, T. and S. Coate (1998) "Sources of Inefficiency in a representative Democracy: A dynamic Analysis", American Economic Review, 88(1): 139-156. 
[3] Becher M. (2012), "Presidentialism, Parliamentarism and Redistribution", mimeo.

[4] Diermeier, D. and R. Vlaicu (2011), "Executive Control and legislative Success", Review of Economic Studies 78:846-871.

[5] Elgie, R. (2005) "From Linz to Tsebelis: Three Waves of Presidential/Parliamentary Studies?", Democratization, 12(1): 106-122.

[6] Persson, T. and G. Tabellini (2002) Political Economics: Explaining Economic Policy, MIT Press.

[7] Persson, T. and G. Tabellini (2005) The Economics effects of Constitutions, MIT Press.

[8] Vlaicu, R. (2008) "Executive Performance under Direct and Hierarchical Accountability Structures: Theory and Evidence". Available at SSRN: http://ssrn.com/abstract $=1051661$.

[9] Whalley, A. and R. Vlaicu (2013) "Hierarchical Accountability in Government: Theory and Evidence". Available at SSRN: http://ssrn.com/abstract $=1925005$. 


\section{Appendix}

Proof of Proposition 1. Legislators in the presidential system face undistorted incentives. Policy-oriented legislators approve $B$ only when it is efficient, i.e. when $s_{t}=s_{B}$. As the vote of the parliament does not influence who is in office in the second period, office-oriented legislators in both periods maximise their utility by maximising the reputation of the parliament, i.e. by mimicking the behaviour of the policy-oriented ones. As a consequence the parliament approves $B$ only when $s_{t}=s_{B}$.

In the second period if $s_{t}=s_{A}$ both types of executive are indifferent between offering $A$ and $B$, as in either case the parliament induces the implementation of $A$. If $s_{t}=s_{B}$ both types offer $B$, which is approved, and nobody gains by deviating to $A$ because a policy-oriented executive would have both a lower utility from the policy and lower rents, and an office-oriented would have lower rents.

Given the second period behaviour the same considerations apply to the first period strategies. No type can gain from deviating, because the assembly votes in a way that the efficient policy is implemented and voters observe only $g_{t}$, so no deviation can induce a gain in reputation.

Proof of Proposition 2. The second period behaviour is the same in the two equilibria. Legislators in the second period face the same undistorted incentives as in the presidential system, hence both types of legislators approve $B$ only when $s_{2}=s_{B}$. Given the efficient behaviour of the assembly what matters for the executive is the reputation; as voters in the parliamentary system observe both the proposed and the implemented policy both types of executive find optimal to propose the efficient policy $g^{*}\left(s_{t}\right)$.

In the first period the parliament faces a distorted incentive scheme. In particular, while policy-oriented legislators find still optimal to vote for the efficient policy (i.e. approve $B$ only when $s_{1}=s_{B}$ ), office-oriented legislators find optimal to approve any policy proposal so to be in power in period 2.

In the first period in Equilibrium 1 a policy-oriented executive never gains from deviation, since there's no gain in utility nor reputation. An office-oriented executive could instead gain by deviating and offering $A$ when $s_{t}=s_{A}$; for this not to be a profitable deviation the following must hold:

$$
1-\Gamma c_{A}+(1-\Gamma)\left(1-\frac{1}{2} c_{A}\right)+\varepsilon(0)>1-c_{A}+\left(1-\frac{1}{2} c_{A}\right)+\varepsilon(1),
$$


which is satisfied iff $c_{A}>\frac{2(\varepsilon+\Gamma)}{2-\Gamma}$.

In the first period in Equilibrium 2 a policy-oriented executive never gains from deviation, as again there's no gain in utility nor reputation. An office-oriented executive could instead gain by deviating and offering $B$ when $s_{t}=s_{A}$; for this not to be a profitable deviation the following must hold:

$$
1-c_{A}+\left(1-\frac{1}{2} c_{A}\right)+\varepsilon(\gamma)>1-\Gamma c_{A}+(1-\Gamma)\left(1-\frac{1}{2} c_{A}\right)+\varepsilon(0),
$$

which is satisfied iff $c_{A}<\frac{2(\varepsilon \gamma+\Gamma)}{2-\Gamma}$.

Proof of Proposition 3. Let $\widehat{\gamma}^{p}$ be the updated belief that the legislators have on the policy motivation of the executive at the beginning of period two, and $\widehat{\gamma}^{v}$ the updated belief of the voters. Notice that $\widehat{\gamma}^{p}$ is relevant to determine the voting behaviour of the legislators in period 2, while $\widehat{\gamma}^{v}$ is relevant to determine the executive's reputation incentives. Moreover in the presidential system the two beliefs may differ, given that $\widehat{\gamma}^{p}$ is an update of $\gamma$ based on $g_{1}^{e}$ and $s_{1}$, while $\widehat{\gamma}^{v}$ is an update of $\gamma$ based on $g_{1}$ and $s_{1}$, and in general $g_{1}$ may differ from $g_{1}^{e}$. Recall that we assume $\gamma=\frac{1}{2}$.

Second period. Notice that a policy-oriented legislator maximises his utility by voting for what he believes to be the efficient policy given the executive's proposal and equilibrium strategy, while an office-oriented legislator maximises his utility by behaving as a policy-oriented one in order to maximise his end of period reputation. Given the second period executive's equilibrium behaviour, if the parliament observes $g_{2}^{e}=B$ and $\sigma_{2}=s_{B}$ it approves $B$ because the signal that the legislators receive is compatible with the policy that is proposed by the executive. If the parliament observes $g_{2}^{e}=B$ and $\sigma_{2}=s_{A}$, instead, it computes $\operatorname{Pr}\left[s_{2}=s_{B} \mid g_{2}^{e}=B, \sigma_{2}=s_{A}\right]$ in order to decide on its vote. Such probability, given the equilibrium strategies, is

$$
\begin{aligned}
\operatorname{Pr}\left[s_{2}=s_{B} \mid g_{2}^{e}=B, \sigma_{2}=s_{A}\right] & =\frac{\operatorname{Pr}\left[g_{2}^{e}=B, \sigma_{2}=s_{A} \mid s_{2}=s_{B}\right] \cdot \operatorname{Pr}\left[s_{2}=s_{B}\right]}{\operatorname{Pr}\left[g_{2}^{e}=B, \sigma_{2}=s_{A}\right]} \\
& =\frac{1-\rho}{1-\widehat{\gamma}^{p} \rho}=\frac{1}{3-2 \widehat{\gamma}^{p}}
\end{aligned}
$$

the parliament approves $B$ after $\sigma_{2}=s_{A}$ iff $\operatorname{Pr}\left[s_{2}=s_{B} \mid g_{2}^{e}=B, \sigma_{2}=\right.$ $\left.s_{A}\right]>\frac{1}{2}$, which happens when $\widehat{\gamma}^{p}>\frac{1}{2}$. We assume that legislators 
follow their signal when indifferent. Based on the equilibrium strategies, and on the voting behaviour of the parliament the beliefs of the parliament at the beginning of period 2 are:

$$
\begin{aligned}
& \widehat{\gamma}^{p}\left(A, s_{A}\right)=\operatorname{Pr}\left(\theta^{e}=1 \mid g_{1}^{e}=A, s_{1}=s_{A}\right)=1, \\
& \widehat{\gamma}^{p}\left(A, s_{B}\right)=\operatorname{Pr}\left(\theta_{e}=1 \mid g_{1}^{e}=A, s_{1}=s_{B}\right)=\frac{1}{2}, \\
& \widehat{\gamma}^{p}\left(B, s_{A}\right)=\operatorname{Pr}\left(\theta^{e}=1 \mid g_{1}^{e}=B, s_{1}=s_{A}\right)=0, \\
& \widehat{\gamma}^{p}\left(B, s_{B}\right)=\operatorname{Pr}\left(\theta^{e}=1 \mid g_{1}^{e}=B, s_{1}=s_{B}\right)=\frac{1}{2} .
\end{aligned}
$$

All the above beliefs are derived by Bayes' rule, apart from $\widehat{\gamma}^{p}\left(A, s_{B}\right)$ which is an out-of-equilibrium belief. We assume that the parliament in this case holds passive beliefs (that also coincide with voters' beliefs) and does not update the executive's reputation.

Voters' beliefs are different from the legislators' beliefs, as voters do not observe the policy proposal $g_{1}^{e}$; hence, they condition to the implemented policy $g_{1}$. Based on the equilibrium strategies, and on the voting behaviour of the parliament the voters' beliefs at the beginning of period 2 are:

$$
\begin{gathered}
\widehat{\gamma}^{v}\left(A, s_{A}\right)=\operatorname{Pr}\left(\theta^{e}=1 \mid g_{1}=A, s_{1}=s_{A}\right)=\frac{\gamma}{\gamma+(1-\gamma) \rho}=\frac{3}{5} \\
\widehat{\gamma}^{v}\left(A, s_{B}\right)=\operatorname{Pr}\left(\theta_{e}=1 \mid g_{1}=A, s_{1}=s_{B}\right)=\frac{1}{2}, \\
\widehat{\gamma}^{v}\left(B, s_{A}\right)=\operatorname{Pr}\left(\theta^{e}=1 \mid g_{1}=B, s_{1}=s_{A}\right)=0 \\
\widehat{\gamma}^{v}\left(B, s_{B}\right)=\operatorname{Pr}\left(\theta^{e}=1 \mid g_{1}=B, s_{1}=s_{B}\right)=\frac{1}{2} .
\end{gathered}
$$

Notice that $\widehat{\gamma}^{v}\left(A, s_{A}\right)<1$, as $g_{1}=A$ could derive both from the executive's efficient behaviour and from a rejection by the parliament of the executive's inefficient proposal. On the other side, $\widehat{\gamma}^{v}\left(A, s_{B}\right)=\frac{1}{2}$ as the outcome is in this case interpreted as a mistaken rejection by the parliament of the efficient policy proposed by the executive.

- Let's consider first the incentives after $s_{1}=s_{B}$. In this case $\widehat{\gamma}^{p}=\frac{1}{2}$ so that legislators follow their signal when it is in contrast with the executive's policy proposal. Notice moreover that in this situation $\widehat{\gamma}^{p}=\widehat{\gamma}^{v}$. The ex-post reputation after $A$ is smaller than 1 , because there are cases in which the executive proposes $B$ and $B$ is not approved so that $g_{2}=A$ can be observed even if the executive is office-oriented. More precisely we have:

$$
\operatorname{Pr}\left[\theta_{e}=1 \mid g_{2}=A\right]=\frac{\operatorname{Pr}\left[g_{2}=A \mid \theta_{e}=1\right] \operatorname{Pr}\left[\theta_{e}=1\right]}{\operatorname{Pr}\left[g_{2}=A\right]}=\frac{(2-\rho) \widehat{\gamma}^{v}}{1+\widehat{\gamma}^{v}-\rho \widehat{\gamma}^{v}}=\frac{4}{7},
$$


and the ex-post reputation after $B$ is

$\operatorname{Pr}\left[\theta_{e}=1 \mid g_{2}=B\right]=\frac{\operatorname{Pr}\left[g_{2}=B \mid \theta_{e}=1\right] \operatorname{Pr}\left[\theta_{e}=1\right]}{\operatorname{Pr}\left[g_{2}=B\right]}=\frac{\rho \widehat{\gamma}^{v}}{1-\widehat{\gamma}^{v}+\rho \widehat{\gamma}^{v}}=\frac{2}{5}$,

where $\operatorname{Pr}\left[\theta_{e}=1 \mid g_{2}=A\right]>\frac{1}{2}>\operatorname{Pr}\left[\theta_{e}=1 \mid g_{2}=B\right]$, so that observing the implemented policy $A(B)$ increases (decreases) the voters' belief on the executive's policy motivation with respect to the belief $\widehat{\gamma}^{v}$ held at the beginning of the second period. Notice that, given the precision of the signal received by the parliament, the expected reputation from proposing $B$ when the state is $s_{2}=$ $s_{A}$ is $\rho \frac{4}{7}+(1-\rho) \frac{2}{5}=\frac{18}{35}$, while the expected reputation from proposing $B$ when the state is $s_{2}=s_{B}$ is $\rho \frac{2}{5}+(1-\rho) \frac{4}{7}=\frac{16}{35}$.

The strategies $g_{2}^{e}\left(s_{2}, 1\right)=g^{*}(s)$ and $g_{2}^{e}\left(s_{2}, 0\right)=B$ constitute a pure strategy NE if no type of executive has incentive to deviate. A type $\theta^{e}=0$ could deviate and choose $g_{2}^{e}\left(s_{A}, 0\right)=A$. For this not to be a profitable deviation it must be:

$$
1-\frac{2}{3} c_{A}+\varepsilon \frac{18}{35} \geq 1-c_{A}+\varepsilon \frac{4}{7},
$$

which is satisfied given that $c_{A}>\varepsilon$. The deviation to $g_{2}^{e}\left(s_{B}, 0\right)=$ $A$ is even less profitable, and hence is avoided by the same condition.

A policy-oriented type never deviates to $g_{2}^{e}\left(s_{A}, 1\right)=B$ as this deviation decreases both the utility from policy implementation and the final reputation. He could however deviate and choose $g_{2}^{e}\left(s_{B}, 1\right)=A$. For this not to be a profitable deviation it must be:

$$
\frac{7}{4}-\frac{1}{3} c_{A}+\varepsilon \frac{16}{35} \geq 1-c_{A}+\varepsilon \frac{4}{7},
$$

which is satisfied because of the assumption $c_{A}>\varepsilon$.

- Let's now consider the incentives after $s_{1}=s_{A}$ and $g_{1}^{e}=g_{1}=B$. In this case $\widehat{\gamma}^{v}=\widehat{\gamma}^{p}=0$ so the ex post reputation of the executive is 0 regardless of the policy that is implemented in the second period. As a consequence, the office-oriented executive has no incentive to deviate from proposing $B$ in every state. A policyoriented executive never proposes $g_{1}^{e}=B$ when $s_{1}=s_{A}$.

- The more complex case to analyse is the case in which $s_{1}=s_{A}$ and $g_{1}=A$, as voters are not able to observe whether the implementation of $A$ originates from $g_{1}^{e}=A$ or $g_{1}^{e}=B$, while the parliament 
can. As a consequence, $\widehat{\gamma}^{v}=\frac{3}{5}$, while $\widehat{\gamma}^{p} \in\{0,1\}$. This may be an issue, as the legislators' behaviour differs according to their belief on the executive's type. Hence the ex-post reputations in this case are

$$
\begin{aligned}
& \operatorname{Pr}\left[\theta_{e}=1 \mid g_{2}=A\right]=\frac{\operatorname{Pr}\left[g_{2}=A \mid \theta_{e}=1\right] \operatorname{Pr}\left[\theta_{e}=1\right]}{\operatorname{Pr}\left[g_{2}=A\right]}=\frac{\frac{1}{2} \widehat{\gamma}^{v}}{\frac{1}{2}}=\frac{3}{5}, \\
& \operatorname{Pr}\left[\theta_{e}=1 \mid g_{2}=B\right]=\frac{\operatorname{Pr}\left[g_{2}=B \mid \theta_{e}=1\right] \operatorname{Pr}\left[\theta_{e}=1\right]}{\operatorname{Pr}\left[g_{2}=B\right]}=\frac{\frac{1}{2} \widehat{\gamma}^{v}}{\frac{1}{2}}=\frac{3}{5} .
\end{aligned}
$$

Notice that none of the possible actions has an effect on the final reputation. Hence, none of the executive's types has an incentive to deviate from their equilibrium strategies, as any possible deviation reduces the per-period utility without increasing the final reputation.

First period In the first period a policy-oriented executive offers $g_{1}^{e}\left(1, s_{A}\right)=$ $A$. As discussed above, this implies that the final reputation is $\frac{3}{5}$ regardless of the policy offered in period 2. A policy-oriented executive could deviate and choose instead $g_{1}^{e}\left(1, s_{A}\right)=B$. For $g_{1}^{e}\left(1, s_{A}\right)=B$ not to be a profitable deviation the following must hold:

$$
4-\frac{3}{2} c_{A}+\frac{3}{5} \varepsilon \geq \frac{23}{9}-\frac{7}{9} c_{A} .
$$

The above condition is always satisfied by assumption since $c_{A}<1$.

A policy-oriented executive could also deviate to $g_{1}^{e}\left(1, s_{B}\right)=A$. Both actions induce the same final reputation. Hence, there is no incentive to deviate as the deviation reduces the per period utility without increasing the final reputation.

An office-oriented executive could deviate and choose $g_{1}^{e}\left(0, s_{A}\right)=A$. As discussed above, this deviation implies a final reputation equal to $\frac{3}{5}$; moreover, it implies $\widehat{\gamma}^{p}=1$ and the fact that the parliament approves any policy proposal in the second period. The equilibrium strategy $g_{1}^{e}\left(0, s_{A}\right)=B$, instead, implies a final reputation of $\frac{3}{5}$ if the parliament rejects the first period policy proposal and a final reputation of 0 if it accepts it; hence, it implies an expected final reputation of $\frac{2}{5}$. Moreover, as $\widehat{\gamma}^{p}=0$, it implies that the parliament follows its own 
signal in the second period. For $g_{1}^{e}\left(1, s_{A}\right)=A$ not to be a profitable deviation the following must hold:

$$
2-\frac{7}{6} c_{A}+\frac{2}{5} \varepsilon \geq 2-\frac{3}{2} c_{A}+\frac{3}{5} \varepsilon .
$$

The condition is satisfied given that $c_{A} \geq \varepsilon$.

An office oriented executive could also deviate by choosing $g_{1}^{e}\left(0, s_{B}\right)=A$. As discussed above, when $s_{1}=s_{B}$, both policy proposals induce $\widehat{\gamma}^{v}=$ $\widehat{\gamma}^{p}=\frac{1}{2}$. Hence, this is never a profitable deviation as it decreases the per period profit without any benefit in the second period.

Proof of Proposition 4. In the parliamentary system both $\widehat{\gamma}^{p}$ and $\widehat{\gamma}^{v}$ are updates of $\gamma$ based on $g_{1}^{e}$ and $s_{1}$, hence $\widehat{\gamma}^{v}=\widehat{\gamma}^{p}$. Recall that we assume $\gamma=\frac{1}{2}$, which in turn implies that it is equally likely that the majority of the legislators are policy- or office-oriented $(\Gamma=1 / 2)$. A policy-oriented executive in equilibrium proposes $g_{t}^{e}\left(1, s_{t}\right)=g_{t}^{*}\left(s_{t}\right)$ and an office-oriented one proposes $g_{t}^{e}\left(0, s_{t}\right)=B$.

Policy-oriented legislators, in the first period, are indifferent between following their signal or approving every policy proposal. In equilibrium they follow their signal with probability $x=\frac{15 c_{A}-3}{5 c_{A}+10}$. Office-oriented legislators approve every policy proposal. In the second period policyand office-oriented members of the parliament behave alike: if the executive reputation is 1 they always approve, if it is 0 or $\frac{1}{2}$ they follow their signal $\sigma_{2}$.

Second period In the second period policy-oriented legislators maximise their utility by voting for what they believe to be the efficient policy given the executive's proposal and equilibrium strategy, and officeoriented legislators maximise their utility by behaving as policy-oriented ones, as in the second period of the presidential system. Given the second period executive's equilibrium behaviour, if the parliament observes $g_{2}^{e}=B$ it approves $B$ after $\sigma_{2}=s_{A}$ if $\widehat{\gamma}^{p}>\frac{1}{2}$.

Given the equilibrium strategies, the updated reputation at the beginning 
of period $2, \widehat{\gamma}^{v}=\widehat{\gamma}^{p}=\widehat{\gamma}$ is

$$
\begin{aligned}
& \widehat{\gamma}\left(A, s_{A}\right)=\operatorname{Pr}\left(\theta^{e}=1 \mid g_{1}^{e}=A, s_{1}=s_{A}\right)=1, \\
& \widehat{\gamma}\left(A, s_{B}\right)=\operatorname{Pr}\left(\theta_{e}=1 \mid g_{1}^{e}=A, s_{1}=s_{B}\right)=\frac{1}{2}, \\
& \widehat{\gamma}\left(B, s_{A}\right)=\operatorname{Pr}\left(\theta^{e}=1 \mid g_{1}^{e}=B, s_{1}=s_{A}\right)=0, \\
& \widehat{\gamma}\left(B, s_{B}\right)=\operatorname{Pr}\left(\theta^{e}=1 \mid g_{1}^{e}=B, s_{1}=s_{B}\right)=\frac{1}{2} .
\end{aligned}
$$

All the above beliefs are computed via Bayes' rule, with the exception of $\widehat{\gamma}\left(A, s_{B}\right)$ which is an out-of-equilibrium belief. We assume that in this case voters and legislators hold passive beliefs, and do not update the executive's reputation.

- If $s_{1}=s_{A}$ a policy-oriented executive enters the second period with a reputation $\widehat{\gamma}=1$, and an office-oriented executive enters the second period with a reputation $\widehat{\gamma}=0$. In either case, their final reputation is $\widehat{\gamma}$. Hence an office-oriented executive finds optimal to propose $g_{2}^{e}\left(0, s_{t}\right)=B$, and a policy-oriented executive finds optimal to propose $g_{t}^{e}\left(1, s_{t}\right)=g_{t}^{*}\left(s_{t}\right)$.

- If $s_{1}=s_{B}$ each type of executive enters the second period with reputation $\widehat{\gamma}=\frac{1}{2}$. If the executive proposes $B$ the proposal can be either accepted or rejected by the parliament. As voters observe both the proposed and the implemented policy, the ex-post reputations are as follows:

$$
\begin{aligned}
\operatorname{Pr}\left[\theta^{e}=1 \mid g_{2}^{e}=B, g_{2}=B\right] & =\frac{\operatorname{Pr}\left[g_{2}^{e}=B, g_{2}=B \mid \theta_{e}=1\right] \operatorname{Pr}\left[\theta^{e}=1\right]}{\operatorname{Pr}\left[g_{2}^{e}=B, g_{2}=B\right]} \\
& =\frac{\rho \widehat{\gamma}^{v}}{1-\widehat{\gamma}^{v}+\widehat{\gamma}^{v} \rho}=\frac{2}{5}, \\
\operatorname{Pr}\left[\theta^{e}=1 \mid g_{2}^{e}=B, g_{2}=A\right] & =\frac{\operatorname{Pr}\left[g_{2}^{e}=B, g_{2}=A \mid \theta_{e}=1\right] \operatorname{Pr}\left[\theta^{e}=1\right]}{\operatorname{Pr}\left[g_{2}^{e}=B, g_{2}=A\right]} \\
& =\frac{(1-\rho) \widehat{\gamma}^{v}}{(1-\rho) \hat{\gamma}^{v}+\left(1-\widehat{\gamma}^{v}\right)}=\frac{1}{4}, \\
\operatorname{Pr}\left[\theta^{e}=1 \mid g_{2}^{e}=A\right]= & \frac{\operatorname{Pr}\left[g_{2}^{e}=A \mid \theta_{e}=1\right] \operatorname{Pr}\left[\theta^{e}=1\right]}{\operatorname{Pr}\left[g_{2}^{e}=A\right]}=1 .
\end{aligned}
$$

Notice that the expected reputation when proposing $B$ if $s_{2}=s_{A}$ is $\frac{2}{3} \frac{1}{4}+\frac{1}{3} \frac{2}{5}=\frac{3}{10}$, while the expected reputation when proposing $B$ if $s_{2}=s_{B}$ is $\frac{1}{3} \frac{1}{4}+\frac{2}{3} \frac{2}{5}=\frac{7}{20}$. The strategies $g_{2}^{e}\left(s_{2}, 1\right)=g^{*}\left(s_{2}\right)$ and $g_{2}^{e}\left(s_{2}, 0\right)=B$ constitute a pure strategy NE if no type of executive has incentive to deviate. 
An office-oriented executive could deviate and choose $g_{2}^{e}\left(s_{A}, 0\right)=A$. For this not to be a profitable deviation it must be:

$$
1-\frac{2}{3} c_{A}+\varepsilon \frac{3}{10} \geq 1-c_{A}+\varepsilon
$$

which is satisfied given $\frac{c_{A}}{\varepsilon} \geq 3$. An office-oriented executive could also deviate and choose $g_{2}^{e}\left(s_{B}, 0\right)=A$. For this not to be a profitable deviation it must be:

$$
1-\frac{1}{3} c_{A}+\varepsilon \frac{7}{20} \geq 1-c_{A}+\varepsilon
$$

which is satisfied given $\frac{c_{A}}{\varepsilon} \geq 3$.

A policy-oriented executive could deviate to $g_{2}^{e}\left(1, s_{B}\right)=A$. For this not to be a profitable deviation it must be:

$$
\frac{5}{3}-\frac{1}{3} c_{A}+\varepsilon \frac{7}{20} \geq 1-c_{A}+\varepsilon
$$

which is satisfied given $\frac{c_{A}}{\varepsilon} \geq 3$.

A policy-oriented executive could also deviate to $g_{2}^{e}\left(1, s_{A}\right)=B$. For this not to be a profitable deviation it must be:

$$
2-c_{A}+\varepsilon \geq 1-\frac{2}{3} c_{A}+\varepsilon \frac{3}{10}
$$

which is trivially satisfied given $c_{A}<1$.

First period In the first period the condition $R>\varepsilon$ ensures that an officeoriented legislator prefers to approve any policy in order to remain in power in the second period. Policy-oriented legislators in the first period, given the assumption on $\rho$ and the equilibrium strategies, are indifferent between approving or rejecting $B$ when $\sigma_{1}=s_{A}$; in equilibrium they reject $B$ with probability $x=\frac{15 c_{A}-3}{5 c_{A}+10}$ if $\sigma_{1}=s_{A}$. Recall that $\Gamma=\frac{1}{2}$, given that $\gamma=\frac{1}{2}$.

A policy-oriented executive in equilibrium proposes $g_{1}^{e}\left(1, s_{t}\right)=g_{1}^{*}\left(s_{t}\right)$ and an office-oriented one proposes $g_{1}^{e}\left(0, s_{t}\right)=B$.

An office-oriented executive could deviate and choose $g_{1}^{e}\left(0, s_{B}\right)=A$ or $g_{1}^{e}\left(0, s_{A}\right)=A$, because this would ensure being in power in period 2 . He has the greatest incentive to deviate when $s_{1}=s_{A}$ because of the higher probability rejection of $B$ and the additional gain in reputation. 
For $g_{1}^{e}\left(0, s_{A}\right)=A$ not to be a profitable deviation the following must hold:

$$
\left(1-\rho \Gamma x c_{A}\right)+(1-\rho \Gamma x)\left(1-\frac{1}{2} c_{A}\right) \geq 2-c_{A}+\varepsilon
$$

that is satisfied as the mixing probability of the policy-oriented legislators, $x=\frac{15 c_{A}-3}{5 c_{A}+10}$, is determined as to satisfy the above constraint with equality.

A policy-oriented executive could deviate and choose $g_{1}^{e}\left(1, s_{B}\right)=A$. Notice that $\widehat{\gamma}\left(A, s_{B}\right)=\frac{1}{2}=\widehat{\gamma}\left(B, s_{B}\right)$, hence the second period differs only in terms of the probability of reaching it. In particular the expected final reputation is $\frac{1}{2} * 1+\frac{1}{2} * \frac{7}{20}=\frac{27}{40}$. The probability of being in power in the second period is one if the executive proposes $A$ and $1-(1-\rho) \Gamma x$ if the executive proposes $B$ as he is voted out of office only by a parliament with a majority of policy-oriented legislators and an incorrect signal. Hence, for $g_{1}^{e}\left(1, s_{B}\right)=A$ not to be a profitable deviation the following must hold:

$$
\begin{aligned}
& 1+(1-(1-\rho) \Gamma x)-(1-\rho) \Gamma x c_{A} \\
& +(1-(1-\rho) \Gamma x)\left(1+\frac{1}{2}\left(1-c_{A}+\rho-(1-\rho) c_{A}\right) \varepsilon \frac{27}{40}\right) \\
\geq & 1-c_{A}+1+\frac{1}{2}\left(1-c_{A}+\rho-(1-\rho) c_{A}+\varepsilon \frac{27}{40}\right)
\end{aligned}
$$

which given our assumptions on $\varepsilon$, and given $x=\frac{15 c_{A}-3}{5 c_{A}+10}$, is satisfied for any possible value of $c_{A}$. 